\title{
Bay-Region-Selective Annulative $\pi$-Extension (APEX) of Perylene Diimides with Arynes
}

\author{
Takayuki Nakamuro ${ }^{\mathrm{a}, 1}$ (D) \\ Kazushi Kumazawaa \\ Hideto Ito*a,b (D) \\ Kenichiro Itami*a,b,c (D) \\ a Graduate School of Science, Nagoya University, Chikusa, \\ Nagoya 464-8602, Japan \\ ito.hideto@g.mbox.nagoya-u.ac.jp \\ bST-ERATO, Itami Molecular Nanocarbon Project, Nagoya \\ University, Chikusa, Nagoya 464-8602, Japan \\ ' Institute of Transformative Bio-Molecules (WPI-ITbM), Nagoya \\ University, Chikusa, Nagoya 464-8602, Japan \\ itami@chem.nagoya-u.ac.jp \\ Published as part of the 30 Years SYNLETT - Pearl Anniversary Issue
}
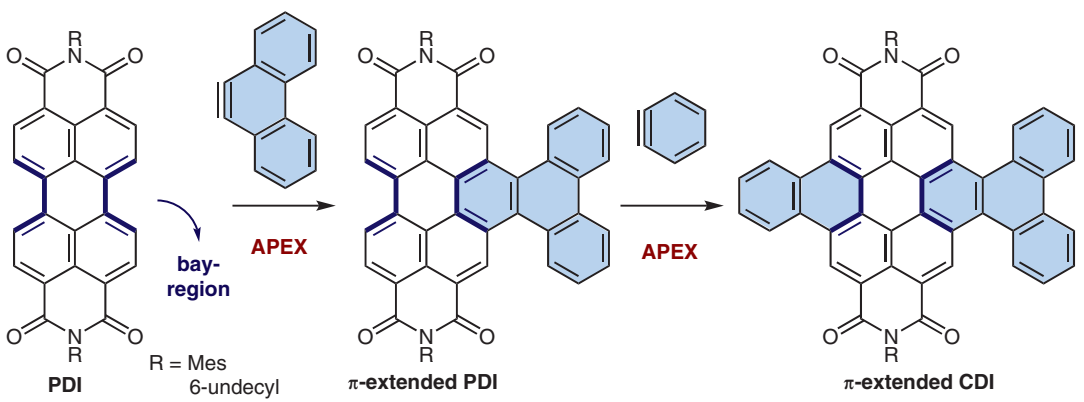

Bay-region-selective annulative $\pi$-extension (APEX)

- One-step $\pi$-extension at unfunctionalized bay-region

- No halogenation/oxidation

- Two-directional APEX

- 5 examples of $\pi$-extended PDI and CDI with moderate yields
Received: 30.11.2018

Accepted after revision: 10.01.2019

Published online: 07.02 .2019

DOI: 10.1055/s-0037-1611668; Art ID: st-2018-b0780-

License terms: cc)

Abstract $A$ bay-region-selective annulative $\pi$-extension (APEX) reaction of perylene diimides (PDIs) has been achieved by means of in-situ generated reactive aryne intermediates. This method provides an efficient one-pot $\pi$-extension at the short axis of PDIs in a sequential manner. Mechanistically, an inverse-electron-demand Diels-Alder reaction might be operative for the transformation.

Key words annulative $\pi$-extension, perylene diimides, arynes, DielsAlder reaction, coronene diimides, polycyclic aromatic hydrocarbons

Perylene diimides (PDIs) ${ }^{2}$ are useful chemical scaffolds because of their rigid perylene $\pi$-conjugated systems and their electron-withdrawing imide groups. These unique $\pi-$ systems bestow attractive optoelectronic properties ${ }^{3}$ that have contributed to versatile applications, for example, as fluorescent materials, ${ }^{3 a}$ as laser compositions, ${ }^{3 b}$ and even in supramolecular chemistry. ${ }^{3 \mathrm{c}}$ Therefore, a variety of PDI derivatives, together with methods for their functionalization, have been developed in attempts to improve and/or modify their optoelectronic and self-assembling properties. ${ }^{4}$ The $\pi$ character of PDIs can be modulated by $\pi$-extensions of PDI in two ways: along the longitudinal (long-axis) ${ }^{5}$ or along the transverse (short-axis) ${ }^{6}$ molecular axis. Although longitudinal modifications of PDIs have been well studied for the development of near-infrared-absorbing materials, the corresponding transverse extensions are less developed due to difficulties in synthesis. Müllen and co-workers demonstrated a $\pi$-extension of PDIs in the bay-regions (the transverse concave armchair edges) through several steps, in- cluding bromination, Suzuki-Miyaura coupling, and cyclization [Scheme 1(a)]. ${ }^{6 a}$ As demonstrated by other researchers, lateral $\pi$-extension of PDIs relies on stepwise halogenation, coupling reactions, and cyclization or oxidation reactions, which reduces the synthetic efficiency and availability of the $\pi$-extended PDIs and loses the opportunity for further $\pi$-extension and functionalization in later stages of the process. Therefore, the development of direct and step-economical methods for obtaining $\pi$-extended PDIs is in high demand.

Recently, the annulative $\pi$-extension $\left(\right.$ APEX) ${ }^{7}$ reaction has attracted much interest in relation to the synthesis of polycyclic aromatic compounds (PACs), because the APEX reaction permits a one-step $\pi$-extension of nonfunctionalized polycyclic aromatic hydrocarbons (PAHs) or heteroaromatics without any prior functionalization, such as halogenation, thereby providing a variety of nanographenes ${ }^{8}$ or heteroatom-containing PACs ${ }^{9}$ that are difficult to access by conventional methods. For example, we have developed $K$ region (concave armchair edge)-selective APEX reactions of PAHs such as phenanthrenes, ${ }^{8 a}$ pyrenes, ${ }^{8 b}$ corannulene, ${ }^{8 c}$ and chrysene, ${ }^{8 \mathrm{~d}}$ catalyzed by cationic palladium complexes. In addition, APEX reactions of PAHs in the bay-region ${ }^{10}$ have also been demonstrated by Clar and Zander, ${ }^{10 a, b}$ and by the groups of Scott, ${ }^{10 \mathrm{c}-\mathrm{f}, \mathrm{j}} \mathrm{Wu},{ }^{10 \mathrm{~g}}$ Matsuda and Stork, ${ }^{10 \mathrm{~h}, \mathrm{i}}$ Kubo, ${ }^{10 \mathrm{k}}$ Peña, ${ }^{101}$ and Hoye. ${ }^{10 \mathrm{~m}}$ These reactions involve Diels-Aldertype reactions with dienophiles such as alkynes, quinones, or arynes [Scheme 1(b)]. However, the range of available substrates is limited to perylene, benzoperylene, and bisanthene, exclusively, due to the harsh reaction conditions, and no examples of bay-region-selective APEX of PDIs have been reported despite the high demand for such processes. 
(a) $\pi$-extension of perylene diimides

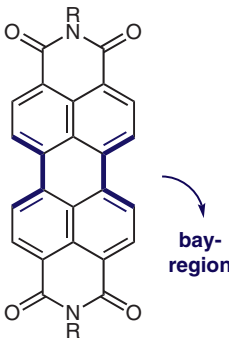

PDI
Classical $\pi$-extension

(i) bromination

(ii) Suzuki-Miyaura coupling

(iii) cyclization

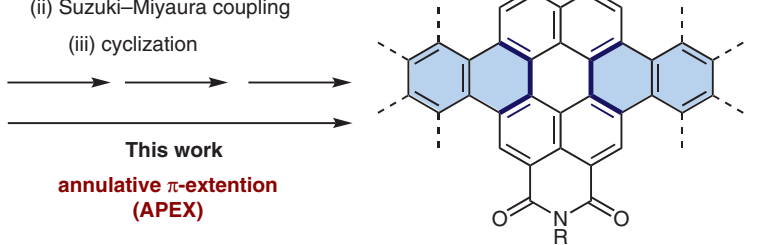

$\pi$-extended PD

(b) APEX reactions of perylene with arynes (Stork/Matsuda, Scott, Kubo, Peña, Hoye) $)^{10}$<smiles>c1cc2cccc3c4cccc5ccc6cccc(c(c1)c23)c4c65</smiles>

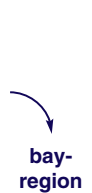

annulative $\pi$-extention (APEX)

Scheme 1 (a) The classical stepwise $\pi$-extension method and the bay-region-selective annulative $\pi$-extension (APEX) method (this work) for $\pi$-extended perylene diimides. (b) Previously developed APEX reaction of perylene with arynes.

Table 1 Screening of the reaction conditions for bay-region-selective APEX reactions of perylene diimide 1 with benzyne precursors $\mathbf{2}^{\text {a }}$
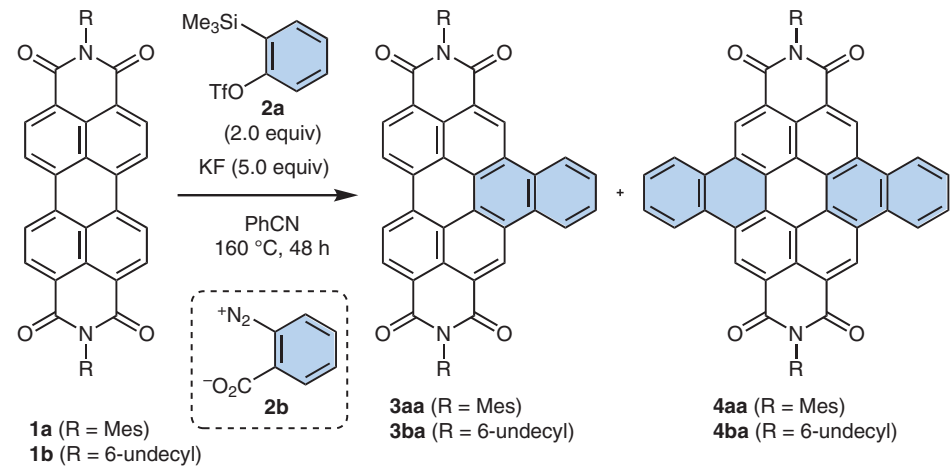

\begin{tabular}{|c|c|c|c|}
\hline Entry & Deviations from the standard conditions for the reaction of $\mathbf{1} \mathbf{a}$ with $\mathbf{2 a}$ & Yield $^{\mathrm{b}}(\%)$ of $3 a a$ & Yield ${ }^{\mathrm{b}}(\%)$ of $4 \mathrm{aa}$ \\
\hline 1 & - & $63(37)^{c}$ & 13 \\
\hline 2 & $120^{\circ} \mathrm{C}$ & 55 & 10 \\
\hline 3 & $24 \mathrm{~h}$ & 58 & 9 \\
\hline 4 & $2 \mathbf{2 a}$ (5.0 equiv) & 38 & 25 \\
\hline 5 & $\mathbf{2 b}$ instead of $\mathbf{2 a}$ & 24 & n.d. ${ }^{d}$ \\
\hline 6 & $\mathrm{MeCN}$ instead of PhCN & trace & n.d. \\
\hline 7 & DMF instead of $\mathrm{PhCN}$ & n.d. & n.d. \\
\hline 8 & toluene instead of $\mathrm{PhCN}$ & n.d. & n.d. \\
\hline 9 & CsF instead of $\mathrm{KF}$ & 35 & trace \\
\hline 10 & TBAT instead of KF & 13 & trace \\
\hline 11 & $\mathbf{1 b}$ instead of $\mathbf{1 a}$ & $68(46)^{\mathrm{e}}$ & $9^{f}$ \\
\hline
\end{tabular}

a Standard reaction conditions: 1 a $\left(0.10 \mathrm{mmol}, 1.0\right.$ equiv), $2 \mathrm{a}$ (2.0 equiv), $\mathrm{KF}\left(5.0\right.$ equiv), $\mathrm{PhCN}(2.0 \mathrm{~mL}), 160{ }^{\circ} \mathrm{C}, 48 \mathrm{~h}$.

${ }^{\mathrm{b}}{ }^{1} \mathrm{H}$ NMR yield determined by using $\mathrm{CH}_{2} \mathrm{Br}_{2}$ as an internal standard.

c Isolated yield.

n.d. $=$ not detected.

e ${ }^{1} \mathrm{H}$ NMR yield of $3 \mathrm{ba}$.

${ }^{\mathrm{f}}{ }^{1} \mathrm{H}$ NMR yield of $\mathbf{4 b a}$. 
Here, we report the first bay-region-selective APEX reactions of PDIs with arynes as $\pi$-extending agents for the synthesis of $\pi$-extended PDIs [Scheme 1(a); bottom].

Optimization of the reaction conditions was performed by using the dimesityl-substituted PDI 1a as a standard substrate (Table 1). PDI 1a was treated with the benzyne ${ }^{11}$ precursor 2-(trimethylsilyl)phenyl triflate (2a) (2.0 equiv) and $\mathrm{KF}$ (5.0 equiv) in benzonitrile as the solvent at $160{ }^{\circ} \mathrm{C}$. After 48 hours, 1a was almost consumed ( $>90 \%$ conversion) to afford the single-APEX product 3aa and the double-APEX product 4aa in 63\% and 13\% NMR yields, respectively (Table 1 , entry 1). However, the separation of product 3aa from 4aa was difficult, and 3aa was obtained in only 37\% isolated yield. Reactions at a lower temperature or for a shorter reaction time resulted in slightly lower yields of the product (entries 2 and 3). The amount of $\mathbf{2 a}$ affected the ratio of 3aa and 4aa; however, an exclusive synthesis of the doubly- $\pi$ extended PDI 4aa was not achieved by changing the reaction conditions (entry 4). The use of 2-diazoniobenzoate (2b) instead of $\mathbf{2 a}$ gave an inferior result, probably due to the thermal lability of $\mathbf{2 b}$ (entry 5; 24\% NMR yield of 3aa). An examination of various solvents revealed that benzonitrile is the optimal solvent [for details, see the Supporting Information (SI)]. In reactions using CsF or tetrabutylammonium difluorotriphenylsilicate (TBAT) instead of $\mathrm{KF}$, 3aa was exclusively obtained, but in a lower yields of 35\% and 15\%, respectively (entries 9 and 10); none of the doubleAPEX product 4aa was generated. The reaction of PDI $\mathbf{1 b}$, which had 6-undecyl groups on the nitrogen atoms, was also examined, and comparable results to those for 1a were obtained (entry 11).

Next, we explored the substrate scope of arynes in the bay-region-selective APEX reactions of PDIs $\mathbf{1 a}$ and $\mathbf{1 b}$ (Scheme 2). When the commercially available 2,3-naphthalyne precursor $\mathbf{2 c}$ was subjected to the APEX reaction with 1a under the optimized reaction conditions shown in entry 1 of Table 1, only traces of the desired APEX product 3ac were observed in the crude mixture. This situation was not markedly improved by changing various reaction conditions, such as the temperature, the amounts of reagents, or the solvent. This might be due to the instability of the 2,3naphthalyne intermediate under severe thermal reaction conditions. ${ }^{12}$ However, the reaction of the highly soluble 6undecyl-substituted PDI 1b proceeded smoothly in MeCN at $120{ }^{\circ} \mathrm{C}$ to afford the APEX product 3bc in 49\% NMR yield and $36 \%$ isolated yield. On the other hand, the 9,10-phenanthryne precursor $\mathbf{2 d}$ reacted with PDIs $\mathbf{1 a}$ and $\mathbf{1 b}$ with good efficiency to give the corresponding single-APEX products 3ad and 3bd, respectively. In these reactions, although traces of the corresponding double-APEX products were also detected in the crude mixture, products 3bc, 3ad, and 3bd were easily isolated by simple chromatography techniques. When other arynes, such as electron-deficient 2,3-pyri- dyne $^{13}$ or alkyl- or aryl-substituted alkynes were subjected to the above reaction conditions, the starting PDIs were recovered without the formation of any APEX products. These results suggest that highly reactive triple bond, such as those of aromatic-hydrocarbon-based arynes are necessary for bay-region-selective APEX reaction of PDIs (see SI for details).

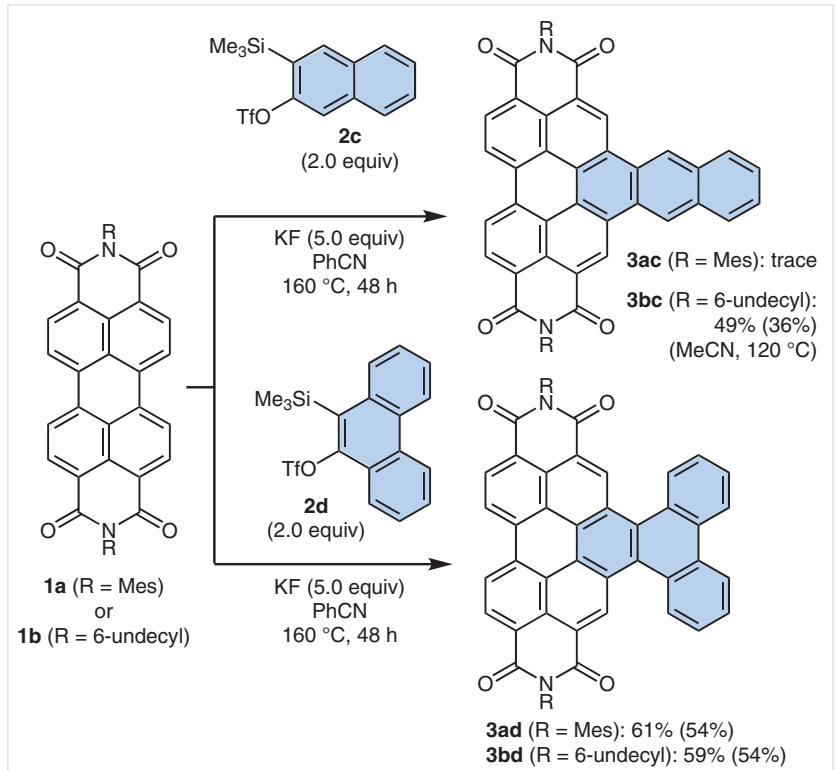

Scheme 2 Substrate scope of arynes in APEX reactions of PDIs 1 a and 1b. Standard reaction conditions: $\mathbf{1 a}(0.050 \mathrm{mmol}, 1.0$ equiv), $\mathbf{2 a}(2.0$ equiv), $\mathrm{KF}$ (5.0 equiv), $\mathrm{PhCN}(1.0 \mathrm{~mL}), 160^{\circ} \mathrm{C}, 48 \mathrm{~h}$. ${ }^{1} \mathrm{H}$ NMR yields were determined by using $\mathrm{CH}_{2} \mathrm{Br}_{2}$ as an internal standard. Isolated yields are shown in parentheses.

To clarify the reaction profiles and to explore the potential of our bay-region APEX reaction, we performed sequential APEX reactions (Scheme 3). First, the single-APEX product 3aa was further subjected to the optimized APEX reaction conditions with $\mathbf{2 a}$ in an attempt to obtain the doubleAPEX product 4aa. However, a quite-low conversion of 3aa and the formation of a small amount of 4aa were observed [Scheme 3(a)], which is inconsistent with the result obtained in entry 1 of Table 1 . This result implies that the actual intermediate for the second bay-region APEX reaction in entry 1 of Table 1 might not be 3aa, but instead 3aa- $\mathbf{H}_{2}$, the primary product of the Diels-Alder reaction before the ejection of two hydrogen atoms that completes the APEX reaction through rearomatization. On the contrary, the APEX reaction of 3 ad with $\mathbf{2 a}$ afforded the $\pi$-extended PDI $\mathbf{5}$, along with a good recovery of 3ad [Scheme 3(b)]. The resulting nonsymmetrical nanographene diimide structure might provide a new entry to $\pi$-extended coronene diimides (CDIs) for future photophysical and electrochemical applications. $^{14}$ 
(a)

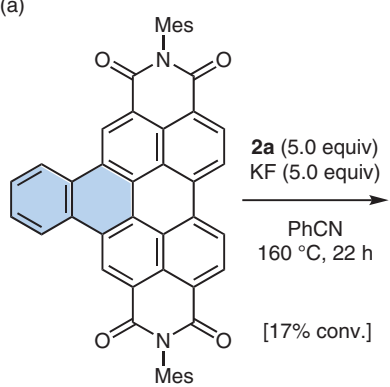

3aa

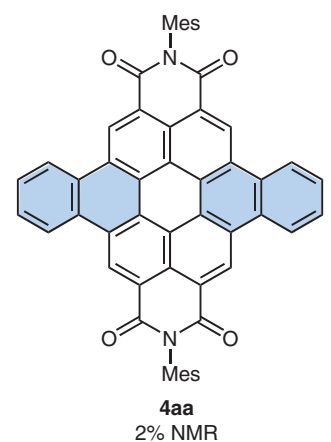

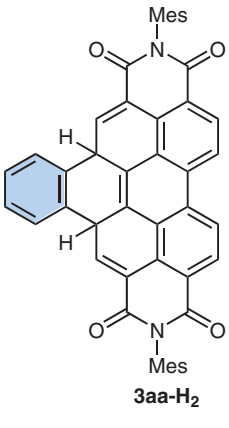

(b)
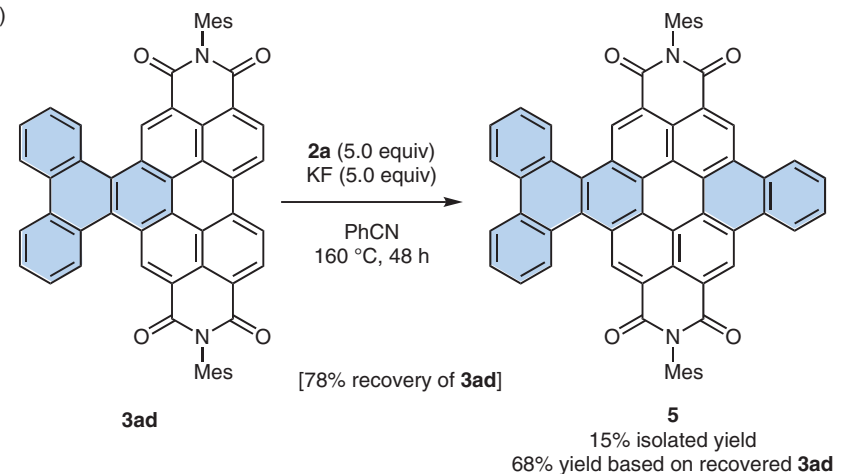

Scheme 3 APEX reactions of 3aa and 3ad for the synthesis of further $\pi$-extended PDIs $\mathbf{4 a a}$ and $\mathbf{5}$

Stimulated by these difference in reactivities between PDIs in the bay-region-selective APEX reaction, we performed DFT calculations at the B3LYP/6-31G(d) level of theory. Scott and co-workers reported that the bay-regionselective APEX reaction of unsubstituted PAHs such as phenanthrene, perylene and bisanthene with acetylene occurs through a Diels-Alder reaction with subsequent aromatization. ${ }^{10 \mathrm{e}}$ Furthermore, they showed experimentally and computationally that perylene is the smallest PAH that affords the APEX product, with an activation energy of 30.0 $\mathrm{kcal} / \mathrm{mol}$ for the Diels-Alder reaction step. In our calcula- tions using PDIs 1a, 3aa, and 3ad as dienes with benzyne as a dienophile, inverse-electron-demand Diels-Alder reactions ${ }^{15}$ successfully afford the dihydro-APEX products 3aa$\mathbf{H}_{\mathbf{2}}$, 4aa- $\mathbf{H}_{\mathbf{2}}$, and 5- $\mathbf{H}_{\mathbf{2}}$ through transition states TS1-TS3, respectively (Scheme 4). The activation barrier to TS1 was calculated to be $14.9 \mathrm{kcal} / \mathrm{mol}$, which is relatively low and supports the smooth reaction progress observed in Table $1 .{ }^{16}$ On the contrary, the activation energies from 3aa and 3ad to TS2 and TS3 were calculated to be 17.1 and 15.7 $\mathrm{kcal} / \mathrm{mol}$, respectively, probably due to the increased LUMO energy of 3aa $(-3.24 \mathrm{eV})$ and $\mathbf{3 a d}(-3.16 \mathrm{eV})$ compared with

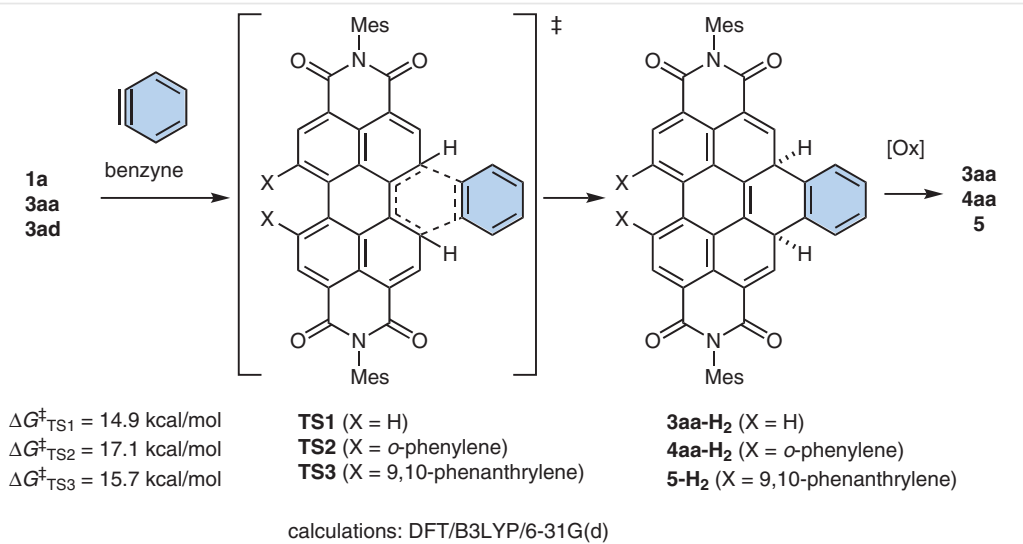

Scheme 4 DFT calculation of the activation energies in Diels-Alder reaction of 1a, 3aa, and 3ad with benzyne 
that of $1 \mathbf{a}(-3.42 \mathrm{eV})$. Although the reason for the lower activation energies in TS3 than TS2 is unclear, the present calculations well reflect the experimental results that DielsAlder reactions occur not in 3aa, but also in 1a and 3ad. Further tuning of arynes (HOMO) and PDIs (LUMO) should lead to even-more-efficient Diels-Alder APEX reactions at nonfunctionalized bay-regions of PDIs.

In summary, we have demonstrated the first bay-region-selective APEX reaction of PDIs by Diels-Alder reactions with arynes. ${ }^{17}$ This method gave the transversally $\pi-$ extended PDIs in a single operation from unfunctionalized PDIs. Even nonsymmetrical $\pi$-extended PDIs, which are difficult to synthesize by conventional methods, were obtained with high efficiency. DFT calculations suggested that the bay-region APEX reaction probably proceeds through an inverse-electron-demand Diels-Alder reaction with a barrier of about $14.9-15.7 \mathrm{kcal} / \mathrm{mol}$. We believe that our APEX method will provide an alternative synthetic tool for preparing various $\pi$-extended PDIs, thereby accelerating research in PDI-based materials science.

\section{Funding Information}

This work was supported by the ERATO program from JST (JPMJER1302 to K.I.), JSPS KAKENHI Grants 18J01322 to T.N. and JP26810057, JP16H00907, JP17K19155, and JP18H02019 to H.I., the SUMITOMO Foundation (141495 to H.I.), and the DAIKO Foundation (H.I.)

\section{Acknowledgment}

The computations were performed at the Research Center for Computational Science, Okazaki, Japan. ITbM, which is supported by the World Premier International Research Center Initiative (WPI), Japan.

\section{Supporting Information}

Supporting information for this article is available online at https://doi.org/10.1055/s-0037-1611668.

\section{References and Notes}

(1) Current address: Graduate School of Science, The University of Tokyo, Hongo, Tokyo 113-0033, Japan.

(2) For a review on perylene diimides, see: Chen, L.; Li, C.; Müllen, K. J. Mater. Chem. C 2014, 2, 1938.

(3) (a) Soh, N.; Ueda, T. Talanta 2011, 85, 1233. (b) Ramírez, M. G.; Morales-Vidal, M.; Navarro-Fuster, V.; Boj, P. G.; Quintana, J. A.; Villalvilla, J. M.; Retolaza, A.; Merino, S.; Díaz-García, M. A. J. Mater. Chem. C 2013, 1, 1182. (c) Würthner, F.; Saha-Möller, C. R.; Fimmel, B.; Ogi, S.; Leowanawat, P.; Schmidt, D. Chem. Rev. 2016, 116, 962. (d) Kojima, M.; Tamoto, A.; Aratani, N.; Yamada, H. Chem. Commun. 2017, 53, 5698. (e) Nagarajan, K.; Mallia, A. R.; Muraleedharan, K.; Hariharan, M. Chem. Sci. 2017, 8, 1776.
(4) (a) Battagliarin, G.; Li, C.; Enkelmann, V.; Müllen, K. Org. Lett. 2011, 13, 3012. (b) Sun, J.; Zhong, F.; Zhao, J. Dalton Trans. 2013, 42, 9595. (c) Ito, S.; Hiroto, S.; Shinokubo, H. Chem. Lett. 2014, 43, 1309.

(5) (a) Pschirer, N. G.; Kohl, C.; Nolde, F.; Qu, J.; Müllen, K. Angew. Chem. Int. Ed. 2006, 45, 1401. (b) Zhao, X.; Xiong, Y.; Ma, J.; Yuan, Z. J. Phys. Chem. A 2016, 120, 7554.

(6) (a) Avlasevich, Y.; Müller, S.; Erk, P.; Müllen, K. Chem. Eur. J. 2007, 13, 6555. (b) Li, Y.; Xu, L.; Liu, T.; Yu, Y.; Liu, H.; Li, Y.; Zhu, D. Org. Lett. 2011, 13, 5692. (c) Chaolumen Enno, H.; Murata, M.; Wakamiya, A.; Murata, Y. Chem. Asian. J. 2014, 9, 3136. (d) Calbo, J.; Doncel-Giménez, A.; Aragó, J.; Ortí, E. Theor. Chem. Acc. 2018, 137, 27.

(7) For reviews on APEX reaction of aromatics, see: (a) Ito, H.; Ozaki, K.; Itami, K. Angew. Chem. Int. Ed. 2017, 56, 11144. (b) Ito, H.; Segawa, Y.; Murakami, K.; Itami, K. J. Am. Chem. Soc. 2019, $141,3$.

(8) For our contributions towards APEX reactions of nonfunctionalized PAHs, see: (a) Ozaki, K.; Kawasumi, K.; Shibata, M.; Ito, H.; Itami, K. Nat. Commun. 2015, 6, 6251. (b) Yano, Y.; Ito, H.; Segawa, Y.; Itami, K. Synlett 2016, 27, 2081. (c) Kato, K.; Segawa, Y.; Itami, K. Can. J. Chem. 2017, 95, 329. (d) Ozaki, K.; Zhang, H.; Ito, H.; Lei, A.; Itami, K. Chem. Sci. 2013, 4, 3416. (e) Ozaki, K.; Murai, K.; Matsuoka, W.; Kawasumi, K.; Ito, H.; Itami, K. Angew. Chem. Int. Ed. 2017, 56, 1361. (f) Matsuoka, W.; Ito, H.; Itami, K. Angew. Chem. Int. Ed. 2017, 56, 12224. For a definition of the APEX reaction, see: (g) Segawa, Y.; Ito, H.; Itami, K. Nat. Rev. Mater. 2016, 1, 15002.

(9) For selected APEX reactions of nonfunctionalized heteroaromatics, see: (a) Paria, S.; Reiser, O. Adv. Synth. Catal. 2014, 356, 557. (b) Ozaki, K.; Matsuoka, W.; Ito, H.; Itami, K. Org. Lett. 2017, 19, 1930. (c) Kitano, H.; Matsuoka, W.; Ito, H.; Itami, K. Chem. Sci. 2018, 9, 7556.

(10) For selected bay-region selective APEX reactions, see: (a) Clar, E. Ber. Dtsch. Chem. Ges. 1932, 65, 846. (b) Clar, E.; Zander, M. J. Chem. Soc. 1957, 4616. (c) Fort, E. H.; Donovan, P. M.; Scott, L. T. J. Am. Chem. Soc. 2009, 131, 16006. (d) Fort, E. H.; Scott, L. T. J. Mater. Chem. 2011, 21, 1373. (e) Fort, E. H.; Jeffreys, M. S.; Scott, L. T. Chem. Commun. 2012, 48, 8102. (f) Fort, E. H.; Scott, L. T. Angew. Chem. Int. Ed. 2010, 49, 6626. (g) Li, J.; Jiao, C.; Huang, K.-W.; Wu, J. Chem. Eur. J. 2011, 17, 14672. For the utilization of arynes, see: (h) Stork, G.; Leonia, N. J.; Matsuda, K. US 3364274, 1968. (i) Stork, G.; Leonia, N. J.; Matsuda, K. US 3364275, 1968. (j) Fort, E. H.; Scott, L. T. Tetrahedron Lett. 2011, 52, 2051. (k) Konishi, A.; Hirao, Y.; Matsumoto, K.; Kurata, H.; Kubo, T. Chem. Lett. 2013, 42, 592. (1) Schuler, B.; Collazos, S.; Gross, L.; Meyer, G.; Pérez, D.; Guitián, E.; Peña, D. Angew. Chem. Int. Ed. 2014, 53, 9004. (m) Xu, F.; Xiao, X.; Hoye, T. R. Org. Lett. 2016, $18,5636$.

(11) For reviews on benzyne chemistry, see: (a) Takikawa, H.; Nishii, A.; Sakai, T.; Suzuki, K. Chem. Soc. Rev. 2018, 47, 8030. (b) Roy, T.; Biju, T. T. Chem. Commun. 2018, 54, 2580. (c) Shi, J.; Li, Y.; Li, Y. Chem. Soc. Rev. 2017, 46, 1707. (d) Bhojgude, S. S.; Bhunia, A.; Biju, A. T. Acc. Chem. Res. 2016, 49, 1658. (e) Pérez, D.; Peña, D.; Guitián, E. Eur. J. Org. Chem. 2013, 5981.

(12) Cioslowski, J.; Piskorz, P.; Moncrieff, D. J. Am. Chem. Soc. 1998, 120, 1695; and references cited therein.

(13) (a) Saito, N.; Nakamura, K.; Shibano, S.; Ide, S.; Minami, M.; Sato, Y. Org. Lett. 2013, 15, 386. (b) For a review on heterocyclic arynes, see: Goetz, A. E.; Shah, T. K.; Garg, N. K. Chem. Commun. 2015, 51, 34. 
(14) (a) Sanyal, S.; Manna, A. K.; Pati, S. K. J. Phys. Chem. C 2013, 117, 825. (b) Zhang, C.; Shi, K.; Jiajun, X.; Lei, T.; Yan, Q.; Wang, J.-Y.; Pei, J.; Zhao, D. Chem. Commun. 2015, 51, 7144. (c) Yang, M.; Zhou, H.; Li, Y.; Zhang, Q.; Li, J.; Zhang, C.; Zhou, C.; Yu, C. J. Mater. Chem. B 2017, 5, 6572. (d) Paul, S. C.; Cammarata, V. J. Electrochem. Soc. 2018, 165, G116.

(15) For a review on recent inverse-electron-demand Diels-Alder reactions, see: Jiang, X.; Wang, R. Chem. Rev. 2013, 113, 5515.

(16) We also calculated each stationary point and transition state by other basis sets (See SI for details).

(17) 3a; Typical Procedure

A screw-capped glass tube containing a magnetic stirrer bar was charged sequentially with the dimesityl PDI 1a $(100 \mu \mathrm{mol}$, 1.0 equiv, $62.4 \mathrm{mg}$ ), KF (0.51 mmol, 5.0 equiv, $29.5 \mathrm{mg}), \mathrm{PhCN}$ $(2.0 \mathrm{~mL}$ ), and 2-(trimethylsilyl)phenyl triflate $(\mathbf{2 a}, 0.20 \mathrm{mmol}$, 2.0 equiv, $60.0 \mathrm{mg}$ ) under a stream of $\mathrm{N}_{2}$. The mixture was stirred at $160{ }^{\circ} \mathrm{C}$ for $48 \mathrm{~h}$, cooled to r.t., and passed through a short pad of silica gel (eluent: $\mathrm{CHCl}_{3}$ ). The organic solvent was removed under reduced pressure to give a crude mixture that was analyzed by ${ }^{1} \mathrm{H}$ NMR $\left(\mathrm{CDCl}_{3}\right)$ with $\mathrm{CH}_{2} \mathrm{Br}_{2}$ as an internal standard. The residue was the purified by flash column chromatography (silica gel) to afford a mixture of 3aa and 4aa, which was further purified by gel-permeation chromatography to give 3aa as a red solid; yield: $26.4 \mathrm{mg}$ ( $37.7 \mu \mathrm{mol}, 37 \%$ isolated).

${ }^{1} \mathrm{H}$ NMR $\left(400 \mathrm{MHz}, \mathrm{CDCl}_{3}\right): \delta=10.2(\mathrm{~s}, 2 \mathrm{H}), 9.32-9.27(\mathrm{~m}, 2 \mathrm{H})$, $9.24(\mathrm{~d}, J=8.4 \mathrm{~Hz}, 2 \mathrm{H}), 9.10$ (d, $J=8.4 \mathrm{~Hz}, 2 \mathrm{H}), 8.19-8.13(\mathrm{~m}, 2$ H), $7.13(\mathrm{~s}, 4 \mathrm{H}), 2.42(\mathrm{~s}, 6 \mathrm{H}), 2.25(\mathrm{~s}, 12 \mathrm{H}) .{ }^{13} \mathrm{C}$ NMR $(150 \mathrm{MHz}$, $\left.\mathrm{CDCl}_{3}\right): \delta=163.5,163.3,138.8,135.2,134.2,131.1,130.0$, 129.51, 129.47, 129.3, 129.2, 129.0, 128.6, 127.9, 125.1, 124.2, 123.3, 122.9, 122.5, 21.3, 17.9. HRMS (MALDI-TOF): $\mathrm{m} / \mathrm{z}[\mathrm{M}+$ $\mathrm{H}]^{+}$calcd for $\mathrm{C}_{48} \mathrm{H}_{33} \mathrm{~N}_{2} \mathrm{O}_{4}$ : 701.2435; found: 701.2434. 